\title{
Le récit de prison comme témoignage sur l'espace hostile : Barreaux de Mohammed Al Bossaty, Le Baiser de la femme-araignée de Manuel Puig et Le dernier jour d'un condamné de Victor Hugo ${ }^{(*)}$
}

\author{
Mona Saraya \\ Professeur-adjoint \\ Faculté des Lettres- Université du Caire
}

\begin{abstract}
:
This study proposes an interpretative reading of three novels of prison belonging to three different cultures, considered as a testimony on hostile space. These selected novels are placed in the context of a left militancy and they engage different historical periods that are added to the initial frame of the events. In the first phase, we have treated the social and poetical aspect as a testimony on the carceral world. In the second phase, we analyze how the authors organize History and fiction. In conclusion, we have stressed on the mythological dimension of the hostile space : it is the symbol of the primordial cavern considered as the world of shadows.
\end{abstract}

\section{Résumé}

L'objectif de cette étude consiste à proposer une lecture interprétative de trois récits de prison appartenant à trois cultures différentes, envisagés comme témoignage sur l'espace hostile. Ces récits choisis s'inscrivent dans le cadre d'un militantisme de gauche et ils mettent en jeu plusieurs époques historiques qui se greffent sur le cadre initial de l'action. Dans un premier temps, nous avons abordé l'aspect social et poétique comme témoignage sur le monde carcéral. Dans un second temps, nous avons étudié comment se fait l'agencement entre Histoire et fiction. En conclusion, nous avons souligné la dimension mythologique de l'espace hostile: elle est le symbole de la caverne primordiale considérée comme le monde des ombres.

${ }^{(*)}$ Bulletin of the Faculty of Arts Volume 80 Issue 8 October 2020 
رواية السجن باعتبار ها شاهد على المكان العدوانى :

أسوار لمحمد البساطى و قبلة المر أة_العنكبوت لمانويل بويج و آخر يوم في

حياة محكوم عليه بالإعدام لقيكتور هوجو لهوبه

الملخص

تطرح هذه الدر اسة قر اعة تفسيرية لثناث روايات تدخل في إطـار أدب

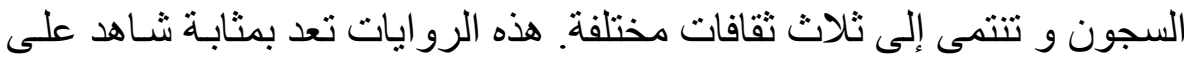

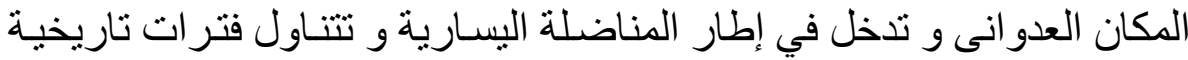

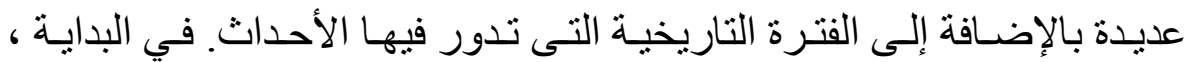

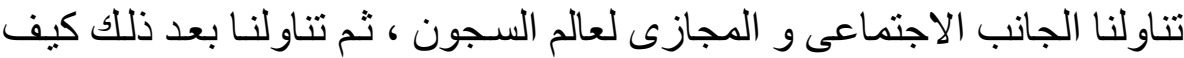

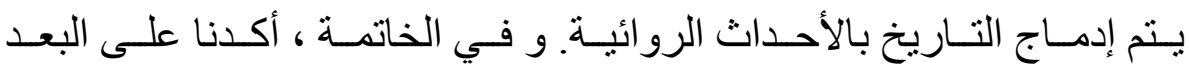

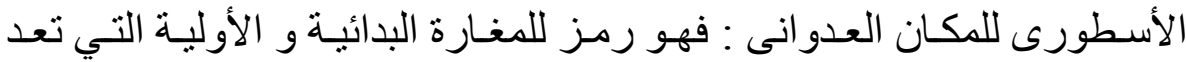
بمثابة عالم الظلايطل

\section{Introduction Introduction}

Le récit de prison est, par nature, un récit hétérogène qui combine plusieurs genres tels que le récit historique et le récit de vie (fictif ou autobiographique). L'objectif de cette étude consiste à proposer une lecture interprétative de trois récits de prison appartenant à trois cultures différentes ; à savoir Barreaux de Mohammed $\mathrm{Al}$ Bossaty, Le Baiser de la femme-araignée de Manuel Puig et Le dernier jour d'un condamné de Victor Hugo. Ces trois récits sont envisagés comme témoignage sur l'espace hostile, ainsi désigné et cité par Bachelard dans La poétique de l'espace. Cet espace hostile donne naissance à des réseaux de sens et d'images qui vont de pair avec les évènements historiques cités dans les récits.

« Nous voulons examiner, en effet, des images bien simples, les images de l'espace heureux. (...) Les espaces d'hostilité sont à peine évoqués dans les pages qui suivent. Ces espaces de la haine et du combat ne peuvent être étudiés qu'en 
se référant à des matières ardentes, aux images d'apocalypse. » 1

C'est ainsi que Bachelard affirme qu'à ses analyses manquait l'étude des espaces d'hostilité ; c'est ce que nous nous proposons d'étudier dans cette recherche.

Le thème de la prison n'est pas propre à ces trois récits choisis. Pour faire une liste non exhaustive des récits de prison dans la littérature internationale, nous en citons :

\section{Classés par ordre de parution pour chaque domaine}

\section{Dans la littérature arabophone :}

$$
\begin{aligned}
& \text { 1. ابن تيمية ، رسائل من السجن ( القرن الرابع عشر بعد الميلاد) }
\end{aligned}
$$

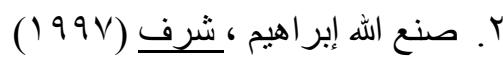

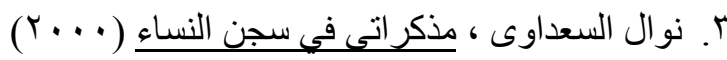

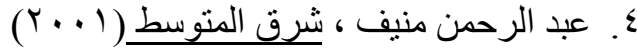

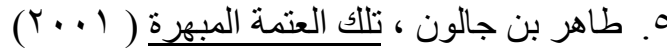

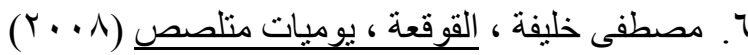

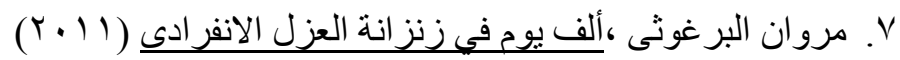

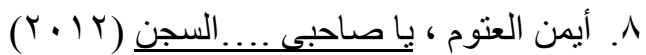

\section{Dans la littérature hispanophone :}

1. Beatriz Esteban, Presas (1997) Prisonnières

2. Carlos Liscano, El furgón de los locos (2001) Le fourgon des fous

3. Mario Condo, Memorias de un preso español (2009) Mémoires d'un prisonnier espagnol

4. Andrés Rabadan, Historias desde la cárcel (2009) Histoires depuis la prison

5. Luis Beldi, Tras los muros. Cárceles. Una historia de locura y corrupción (2013) Derrière les murs. Prisons. Une histoire de folie et de corruption. 


\section{Dans la littérature francophone :}

1. André Malraux, La condition humaine (1933)

2. Albert Camus, L'Etranger (1942)

3. Jean Genet, Le miracle de la rose (1942)

4. Jean-Paul Sartre, Huis Clos (1944)

5. Gérard Etienne, Le nègre crucifié (1971)

6. François Bon, Prison (1998)

7. Malika Oufkir, La prisonnière (2006)

8. Patrick Chamoiseau, La prison vue de l'intérieur (2007)

Le thème de la prison n'est pas propre à la littérature uniquement. En effet, il a fait également l'objet de nombreux tableaux célèbres. Nous en citons quelques tableaux de Goya où il s'agit de l'intérieur de prison, un célèbre tableau de Van Gogh intitulé La ronde des prisonniers (datant de 1890) dont Bossaty se sert pour la couverture de son roman et trois tableaux de Velasquez; à savoir Mujeres en la carcel (Femmes en prison), Encadenada (Enchaînée) et El Ajusticiado (Le supplicié). Mais notre étude portera uniquement sur des récits littéraires, visant ainsi à mettre en évidence leur poétique où l'Histoire et la fiction sont combinés par plusieurs médiums. Cette poétique met en valeur la prison comme archétype primordial du retour aux origines liées aux profondeurs, soulignant ainsi sa dimension mythologique qui va de pair avec l'Histoire et la fiction.

Les thèmes des trois romans choisis pour le corpus de notre étude ont été abordés chacun à part selon plusieurs approches, comme nous allons en donner quelques exemples rapides. Les approches précédentes sont soit littéraires (celle de Marc Olivier Padis et Nada Tomiche), soit interdisciplinaires (entre littérature et peinture : celle de Julia Cuervo Hewitt, et entre littérature et philosophie : celle de Marie 
Naudin) comme nous allons en donner un aperçu dans les lignes qui suivent.

Pour ce qui est de l'approche littéraire, nous citons celle de Marc Olivier Padis et celle de Nada Tomiche. En effet, dans son article intitulé Une littérature de l'enfermement ${ }^{2}$, Marc Olivier Padis aborde la littérature de prison qu'il classe comme genre à part (tout comme la littérature de voyage) où se recoupent plusieurs genres tels que l'autobiographie, le récit historique et le récit d'engagement. Cependant, l'article de Padis ne porte que sur des romans de la littérature française sans citer Le dernier jour d'un condamné. Il en est de même pour Bossaty qui n'a pas été cité dans l'ouvrage de Nada Tomiche intitulé La littérature arabe contemporaine où elle consacre une partie à ce qu'elle appelle l'absurde arabe ${ }^{3}$ (inspiré de l'absurde européen) comme phase de littérature arabophone à laquelle Bossaty appartient. L'approche comparatiste que nous proposons élargira donc le champ d'étude d'un corpus où un même thème est abordé et elle servira à en découvrir d'autres significations. Pour ce qui est des approches interdisciplinaires, nous en citons celle de Julia Cuervo Hewitt. En effet, dans son article intitulé Le texte absent dans Le baiser de la femme-araignée de Manuel Puig : Silences et réticences d'une époque ${ }^{4}$, elle tente d'établir un parallélisme entre Le baiser de la femme-araignée et Las Meninas (le tableau célèbre de Velasquez). Cette tentative se base surtout sur la ressemblance de la structure formelle entre le roman et le tableau. C'est ainsi qu'elle construit des ponts entre littérature et peinture. En outre, le roman de Manuel Puig n'a pas été étudié non plus en comparaison avec une autre œuvre littéraire, mais il a fait l'objet d'une adaptation cinématographique en 1985.

Dans le contexte des approches interdisciplinaires, l'approche que Marie Naudin adopte, de son côté, pour l'analyse de récits de prison a une dimension philosophique (qui va encore plus loin que l'analyse 
littéraire) en rapport avec la question de l'existence. En effet, dans son article intitulé Hugo et Camus face à la peine capitale ${ }^{5}$, elle établit la comparaison entre Le dernier jour d'un condamné et L'Etranger partant de la notion de la peine capitale telle que la présentent les deux auteurs sous la forme d'une fiction.

Pour notre part, nous allons étudier le récit de prison comme témoignage sur l'espace hostile selon les voies proposées par Claudio Guillén et Daniel-Henri Pageaux, deux comparatistes éminents. Nous allons nous atteler à faire le point sur les variations et les invariants comme notions de base en littérature comparée. Guillén souligne l'importance de la dimension historique comme voie de recherche en littérature comparée dans son ouvrage intitulé Entre lo uno y lo diverso; introducción a la literatura comparada (Entre l'Un et le Divers, introduction à la littérature comparée) : «La littérature comparée a été une discipline résolument historique. ${ }^{6}$ De son côté, Pageaux met en valeur l'importance de l'étude du contexte social et historique de l'œuvre littéraire en littérature comparée, partant de la notion d'universalisme : «L'étude des thèmes offre au comparatiste des travaux nombreux, précis : identifier les relations possibles entre les textes et un contexte social, suivre les stratégies discursives, capter les résonances spirituelles et découvrir que l'étude de la littérature peut révéler, comme l'histoire, des dimensions émotionnelles et sensibles. $\gg^{7}$

Présentation des contextes historiques des récits du corpus et justification de ce choix

Georg Lukas souligne, dans son ouvrage intitulé La théorie $d u$ roman, que les conditions socio-historiques et philosophiques de l'œuvre littéraire font partie intégrante de son interprétation :

«Cette transformation des points d'orientation transcendantaux soumet les formes littéraires à une dialectique historico-philosophique qui, selon la nature des divers genres, ne saurait être que variable pour chacun d'entre eux. ${ }^{8}$ 
Il s'agit donc d'une lecture et d'une interprétation sociohistorique du texte envisagé comme exprimant une époque historique déterminée. Dans les récits de prison que nous analysons, l'Histoire occupe une place importante: c'est l'alibi par lequel se fait la description du monde carcéral considéré comme reflétant un autre aspect de l'Histoire souvent passé sous silence, notamment à des périodes d'instabilité politique. Bossaty, Puig et Hugo ont vécu à des périodes de tumultes politiques où la dictature militaire régnait (l'Egypte des années soixante et soixante- dix sous Nasser et Sadat pour Bossaty et l'Argentine des années soixante-dix pour Puig,) et la dictature monarchique de Charles X en France (pour Hugo). Ainsi, leurs récits de prison se placent dans le contexte d'un militantisme de gauche. Au lieu de se limiter au cadre historique des événements narrés, leurs trois récits mettent en jeu plusieurs époques historiques qui se greffent sur le cadre initial de l'action. Avant d'étudier en détails ces récits comme témoignage sur l'espace hostile, nous allons donner un aperçu sur les contextes historiques et biographiques des auteurs dans la mesure où ils jouent un rôle pertinent dans la vision qu'ils présentent du monde carcéral.

Mohammed Al Bossaty (1937-2012) est un écrivain qui appartient à la génération d'intellectuels des années soixante en Egypte $^{9}$. Il s'agit d'une période de grandes transformations politiques, économiques et sociales qui ont eu d'importantes répercussions sur la production littéraire et artistique. En effet, l'atmosphère générale de cette époque citée est une atmosphère lugubre dominée par le profond sentiment de désarroi et de déception suite à la défaite militaire de 1967. C'est dans ce contexte que l'absurde égyptien (inspiré de l'absurde européen) a pris naissance. Il est à noter que cette génération d'intellectuels était constamment en conflit avec l'autorité en place. La production littéraire de cette époque reflète le profond sentiment de désarroi, d'étrangeté et de révolte sur le plan de la forme aussi bien que sur celui du contenu. Les œuvres littéraires datant de cette époque 
ont certaines caractéristiques communes (et que l'on retrouve d'ailleurs dans Barreaux bien que le roman n'ait été publié qu'en 2008). Nous en citons : le refus de la forme romanesque traditionnelle, l'importance accordée au principe de la fragmentation sur tous les plans (narratif, temporel, spatial, etc.), le recours aux techniques du montage littéraire et cinématographique et le traitement des tabous sociaux et politiques. Il s'avère important de souligner également que Bossaty était directeur à l'organisme central de la mobilisation et des statistiques. En effet, il était chargé d'examiner l'état des prisons et de recueillir les plaintes des prisonniers. Ce poste lui a permis de visiter toutes les prisons égyptiennes et c'est ainsi qu'il est le mieux placé pour décrire le monde carcéral de l'intérieur.

De son côté, Manuel Puig ${ }^{10}$ (1932-1990) a vécu lui aussi à époque pleine de troubles politiques dans l'Histoire de l'Argentine. En 1973, à cause de ses activités militantes, il a été exilé de l'Argentine suite aux menaces qui lui ont été adressées par l'Alliance Anticommuniste Argentine désignée aussi par le triple A (qui est une organisation terroriste gérée par le péronisme ${ }^{11}$ de l'extrême droite et des forces armées argentines). Cette alliance séquestrait et tuait les intellectuels et politiciens de gauche considérés comme défendant le marxisme. L'année de la parution du Baiser de la femme-araignée est bien celle du coup d'Etat militaire argentin mené par le général Jorge Rafael Videla contre Estela Martinez de Perón qui était au pouvoir à l'époque. Suite à ce coup d'Etat, Jorge Rafael Videla a pu accéder au pouvoir et mettre fin aux fonctions du Congrès National. De même, il a censuré et réprimé tous les partis politiques. C'est ainsi que cette époque, où la terreur régnait, est marquée par la dictature militaire qui emprisonnait les militants de gauche, tout comme dans l'Egypte de Nasser et de Sadat cités dans Barreaux.

Victor Hugo (1802-1885) était lui aussi politiquement très actif. 12 Il a beaucoup lutté pour l'abolition de la peine de mort. Dans le 
contexte de l'analyse d'un récit de prison, un élément de la biographie de l'auteur ne peut passer sous silence : en effet, alors qu'il n'avait que dix ans, il avait assisté à une exécution publique en Espagne et il en a été profondément affecté de sorte que la peine de mort est devenue une obsession. Suite à ses activités politiques, il a été exilé en Belgique de 1851 à 1870, année de la chute du Second Empire. L'année 1829 (celle de la parution du Dernier jour d'un condamné) est bien celle qui précède la Révolution de 1830 (les Trois Glorieuses) menée contre Charles X qui était au pouvoir depuis 1824 ; c'est ainsi qu'elle a provoqué la chute de la Seconde Restauration (il s'agit de la restauration des Bourbon). La politique de Charles X était basée sur l'absolutisme de l'Ancien Régime, provoquant ainsi la colère du peuple français. D'autre part, il avait dissout la chambre des députés nommés par élection et son règne était surtout marqué par la censure sévère puisqu'il avait imposé des limites à la liberté de la presse et à la liberté de l'expression en général, tout comme la politique de Sadat et Nasser (Egypte) et Rafael Videla (Argentine). En outre, dans la mentalité française en général, la guillotine est liée à la Révolution de 1789 de sorte que le nombre de guillotinés a atteint son comble sous le règne de la Terreur. Il s'agit de la période de la dictature de Robespierre (1793-1794) qui a aboli la monarchie pour fonder la République. En effet, Robespierre faisait guillotiner tous ceux qui s'opposaient à la Révolution. D'ailleurs, dans Le dernier jour d'un jour d'un condamné, Hugo fait dire au condamné une phrase vibrante d'humour noire: «La guillotine est encombrée, et chacun ne doit passer qu'à son tour. » 13

Pour analyser comment ces récits du corpus donnent un témoignage sur le monde carcéral, nous allons aborder dans un premier temps l'aspect social et poétique comme témoignages sur le monde carcéral et, dans un deuxième temps, comment se fait l'agencement entre Histoire et fiction. 


\section{L'aspect social et poétique du monde carcéral}

En principe, tout récit de prison s'articule sur trois instances principales : le prisonnier, l'autorité en place et le gardien (considéré comme étant l'intermédiaire). Le roman de Puig se caractérise par la multiplicité d'instances narratives qui ne se déclarent pas, contrairement à ceux de Bossaty et de Hugo dont la narration est assumée par une seule instance. C'est une instance qui ne cherche pas à se dissimuler puisque ce sont des récits à la première personne, revêtant ainsi un aspect autobiographique. Le baiser de la femmearaignée se compose de deux types principaux de discours : Un discours narratif fictionnel (une histoire principale coupée par des récits de films que Molina raconte) et un discours informatif ${ }^{14}$. Puig fait varier la perspective en présentant les points de vue du prisonnier, de son compagnon de cellule qui est un autre prisonnier mais qui soutire des informations pour le compte de l'autorité, ayant ainsi un double statut, et de l'autorité en place. Puig nous montre ainsi comment chacun d'eux voit les autres. En effet, Puig était particulièrement fasciné par le cinéma et il écrivait des scénarios de films; c'est pourquoi l'écriture cinématographique (qui se base surtout sur les séquences) domine son œuvre. Il a fait des études d'architecture, de philosophie et de cinéma ; c'est ce qui a eu des échos importants sur sa production littéraire, par exemple l'organisation formelle, les différents plans et les différentes perspectives. C'est ainsi que les techniques narratives qu'il utilise ne sont pas purement littéraires.

De son côté, Bossaty se limite à la perspective du gardien qui voit tout. Sa perspective n'est pas extérieure, comme aurait pu le suggérer l'idée. En effet, loin de se restreindre à l'observation à partir du toit de sa maison, il fait entrer le lecteur dans le monde de la prison, tout en dénonçant les tabous sociaux comme l'homosexualité, la perversion sexuelle, le trafic de la drogue (qu'il se chargeait d'apporter aux 
prisonniers) et la corruption. La perspective n'est donc pas celle du prisonnier, contrairement au Dernier jour d'un condamné où Hugo nous montre comment le prisonnier voit l'autorité et le peuple au nom duquel il devrait être exécuté, les autres prisonniers et les gardiens, ne serait-ce que dans quelques épisodes brefs (par exemple l'épisode où un autre prisonnier raconte sa vie au héros). D'autre part, le récit est, la plupart du temps, introspectif puisqu'il se centre sur l'analyse des états d'âmes et sur les réflexions du prisonnier qui en assume la narration. L'introspection apparaît aussi dans Le baiser de la femmearaignée, mais ce n'est que vers la fin du roman où Puig nous fait pénétrer dans la pensée d'Arregui. En effet, il évoque en détails le rêve qu'il fait de la femme-araignée.

Dans ce contexte, les titres sont révélateurs puisqu'ils donnent une indication sur l'élément sur lequel la narration va se centrer. Dans les romans français et argentin, l'intérêt est porté sur l'être humain que la prison abrite alors que dans le roman arabe le titre est consacré à l'élément séparateur et non sur l'être séparé des autres (le prisonnier). En outre, dans Barreaux, le choix de l'élément séparateur montre que la perspective narrative va se centrer sur l'espace de la marginalisation. De son côté, «le baiser» indique un contact entre deux éléments (envisagé en tant qu'élément unificateur, s'opposant ainsi aux barreaux qui séparent) et «l'araignée » qui est le geôlier. Pour ce qui est de Hugo, le titre porte sur une référence temporelle donnant l'idée angoissante d'un compte à rebours, contrairement à Bossaty et à Puig dont les romans ont pour titres des indications spatiales considérées comme symboles de l'aliénation. Notons aussi l'emploi de l'indéfini : Barreaux; l'auteur aurait pu avoir recours à l'article défini et mettre Les. Mais le choix de l'indéfini montre que ces barreaux ne sont pas propres à une époque historique déterminée. Le recours à l'indéfini se retrouve aussi dans le roman français : « un condamné », sans précision, pouvant être n'importe qui puisqu'il s'agit d'une époque où la guillotine était généralisée. 
Si ces trois récits de prison sont racontés à partir de trois perspectives distinctes, celles-ci mettent toutes en valeur un même élément ; à savoir le mauvais traitement que subissent les prisonniers qui sont humiliés. Dans Barreaux, les images de l'agression physique dominent de manière flagrante. Salem parle de détenus agressés physiquement ${ }^{15}$ dont les corps sont tout couverts de sang. En outre, certains prisonniers en agressent d'autres ainsi que les gardiens euxmêmes, y compris Salem (victime d'une agression sexuelle dans son enfance),${ }^{16}$. En effet, six gardiens ont été tués sans qu'on ne sache l'auteur de ces crimes inexplicables. D'autre part, Salem cite l'attitude perfide et hypocrite de l'instance policière chargée de garder la prison. En effet, si les représentants des organisations des droits de l'Homme visitent la prison, l'officier falsifie les demandes des prisonniers et leur montre une cour propre. Par contre, si c'est son directeur qui viendra, les prisonniers sont torturés et il lui fait voir les tâches de sang sur leurs corps. D'autre part, la mauvaise nourriture présentée aux prisonniers est citée dans Barreaux ${ }^{17}$ et dans Le baiser de la femme-araignée où les détenus ont des crises de douleur de l'estomac vu que les plats étaient empoisonnés ${ }^{18}$. De son côté, dans Le dernier jour d'un condamné, le héros de Hugo évoque «le pain noir du cachot, la portion du bouillon maigre puisée au baquet des galériens, être rudoyé, moi qui suis raffiné par l'éducation, être brutalisé des guichetiers et des gardes-chiourme $\gg,{ }^{19}$ tout en soulignant qu'il n'a rien à regretter. D'autre part, il ne manque pas d'évoquer le mauvais traitement que subissent les forçats et les galériens, ${ }^{20}$; c'est ce qui rappelle l'agression physique qu'Arregui subit à la fin du Baiser de la femme-araignée.

Face au traitement inhumain que subissent les prisonniers, dans Barreaux, Salem souligne que les condamnés à mort (qu'il appelle «les rouges » et « les drapeaux du danger ») jouissent d'un traitement bien meilleur que les autres. En effet, on leur accorde une plus grande portion de viande, des sucreries et des cigarettes ${ }^{21}$ et on ne leur 
demande jamais de faire n'importe quel travail forcé. En outre, les prisonniers politiques aident Salem à réviser ses leçons dans son enfance ; c'est pourquoi il sympathise souvent avec eux. De leur côté, dans les romans de Bossaty et de Hugo, les infirmiers sympathisent avec le prisonnier et ils ont pitié de lui (Le dernier jour d'un condamné, ${ }^{22}$, et Le baiser de la femme-araignée, ${ }^{23}$

La perspective narrative (bien qu'elle ne soit pas la même dans les trois romans) souligne l'aspect polyphonique des récits envisagés comme "lieux de convergence de plusieurs voix", tel que les théories de Bakhtine l'affirment dans Esthétique et théorie du roman . ${ }^{24}$

En effet, ces récits de prison se caractérisent par la multiplicité de registres de langue comme témoignage social sur l'Histoire. Bossaty fait alterner langue parlée (ou le dialecte) et langue classique. La langue classique apparaît dans le discours du professeur universitaire détenu ${ }^{25}$ qui donnait des cours de philosophie incompréhensible pour les prisonniers. Son attitude à leur égard ne manque pas d'arrogance: «Ils ne comprendront jamais. Je ne supporte pas les questions idiotes dans mes cours $»^{26}$, tout comme Arregui qui se considérait supérieur parce qu'il était plus cultivé que Molina. Les gardiens s'amusent à se moquer de la langue classique que l'économiste utilise, même en parlant aux autres prisonniers et aux gardiens eux-mêmes. Pour ce qui est de la langue dialectale, elle n'apparaît que dans les dialogues, alors que la langue soutenue apparaît dans la narration linéaire. Cette alternance de codes montre le souci de l'auteur de reproduire le monde des marginalisés tel quel.

Pour sa part, dans Le baiser de la femme-araignée, les boléros chantés par Molina sont le médium par lequel Puig insère la culture populaire (entre autres les variations dialectales). Il s'agit d'un genre musical qui avait pris naissance au XIXème siècle en Amérique Latine. Les paroles sont le plus souvent mélancoliques et elles «contiennent beaucoup de vérité », ${ }^{27}$ tel que le dit Molina. Dans ce 
contexte, il est à noter que la période des dictatures militaires en Amérique Latine (les années trente, quarante et cinquante) est celle de l'apogée de ce genre musical. En effet, les pouvoirs en place encourageaient la floraison de ce genre pour distraire les peuples de sorte qu'ils n'auraient pas le temps de penser à la politique.

Dans Le dernier jour d'un condamné, l'argot comme registre de langue apparait dans la chanson populaire insérée ${ }^{28}$ et dans le discours du prisonnier avec qui le héros a partagé le cachot pour quelques instants ${ }^{29}$. Il est inséré avec des notes explicatives placées en bas de page. Le héros n'hésite pas à lui demander des explications étant donné que ce vocabulaire lui est tout à fait étrange et qu'il désigne comme étant «quelque chose de sale et de poudreux, d'une liasse de haillons que l'on secouerait devant vous. ", ${ }^{30}$. L'on remarque que c'est surtout un langage qui se base sur la métaphore (parfois même l'euphémisme) : par exemple «La sorbonne» pour la tête et « La veuve» pour la guillotine. S'y ajoute l'argot qui apparait dans une chanson que le condamné écoute une jeune fille chanter et il qualifie ses paroles de «monstrueuses», ${ }^{31}$, puisqu'elles reproduisent par l'allusion la situation où se trouve le condamné : «J'ai assassiné un homme et je suis arrêté. »

Outre le thème et le registre de langue dont se sert l'instance narrative pour rendre compte du monde carcéral, elle se sert aussi de la métaphore comme structure sous-jacente qui les reflète, comme nous allons le montrer.

\section{Les instances narratives se servent toutes de la métaphore} pour exprimer la prison. Pour Hugo, la métaphore principale est celle de l'enfer auquel la prison est comparée. En effet, tout le long du roman, un vocabulaire en rapport avec l'enfer domine (horrible, sanglante, fer, cachot). La prison est aussi comparée à un «être horrible», «Je suis sa proie; elle me couve, elle me couve, elle m'enlace de tous ses replis. », ${ }^{32}$ (la répétition est de l'auteur). En 
outre, l'image du gouffre et de la grotte apparaissent dans les rêveries du héros : il imagine un abîme où se rassemblent tous les exécutés qui deviennent des fantômes. Cette image se retrouve aussi dans la pensée obsédante d'Arregui qui rêve d'un long tunnel et d'une mer où il se noie.

Bossaty et Puig privilégient la peinture comme autre médium par lequel le thème de la prison est évoqué par le biais de la métaphore : en effet, ils s'en servent pour la couverture de leurs romans. Bossaty utilise un tableau de Van Gogh intitulé La ronde des prisonniers. Ce tableau a été peint à l'hôpital de Saint Rémy où le peintre a été interné suite à une maladie mentale. Il s'agit d'une copie d'une gravure de Gustave Doré intitulé New Gate exercice yard. Dans le tableau de Van Gogh, on voit des prisonniers qui défilent dans la ronde. Sur leurs visages, on lit une expression lugubre d'abattement. Parmi ces prisonniers, on en voit un qui regarde le spectateur (il s'agit du peintre). D'autre part, dans cette scène de prison, c'est la ronde qui occupe le plan principal alors que les gardiens sont placés à l'écart en position statique. L'idée de la ronde dénote la répétition vaine à l'infini. La scène est un espace fermé et les murs s'élèvent à l'infini ; c'est ce qui empêche de voir le ciel ouvert, donnant ainsi l'image d'un espace d'étouffement. Il est à noter qu'un paragraphe du Dernier jour d'un condamné rappelle ce tableau de Van Gogh dont se sert Bossaty. Il s'agit de la scène où le condamné décrit les prisonniers agrippés aux barreaux et qui sortent vers la cour. Ce sont les forçats que le héros compare à des diables :

«Une foule de visage maigres et blêmes pressés les uns sur les autres, comme les pierres d'un mur et tous pour ainsi dire encadrés dans les entrecroisements des barreaux de fer. C'étaient des prisonniers (...) On eut dit des âmes en peine aux soupiraux du purgatoire qui donne sur l'enfer. (...) brillaient quelques yeux perçants et vifs comme des points de feu. », ${ }^{33}$ 
De son côté, Manuel Puig met comme couverture à son roman un tableau de Maxfield Parrish (1879-1926), qui est un peintre américain dont les tableaux se rapprochent plutôt de la photographie par leur aspect réaliste. Dans le tableau utilisé, on voit une femme qui aspire à la liberté. Mais cette femme est la prisonnière de cheveux, tout comme la proie de l'araignée. De cette image carcérale, une lueur d'espoir se dégage : en effet, le personnage a les cheveux déchainés et les bras ouverts. D'autre part, le fond du tableau est un ciel clair, évoquant lui aussi le thème de la liberté.

De son côté, le tableau dont Hugo se sert pour illustrer le roman met en valeur le tout-dernier moment de la vie du condamné. En effet, on le voit les yeux fermés et tiré vers la guillotine en train de supplier ses bourreaux. On lit sur son visage une expression de peur intense. Ce tableau de couverture évoque la dernière scène du roman où le condamné, sur l'échafaud, demande la grâce à ses bourreaux. Il n'y a aucune lueur d'espoir ni de salvation dans ce tableau, bien que, dans le roman, l'idée d'une possible évasion apparait à plusieurs reprises, laissant sous-entendre un espoir qui plane : d'abord, l'espoir d'obtenir le pourvoi et d'écarter la préméditation ${ }^{34}$, mais dissipé ${ }^{35}$, ensuite l'espoir d'échapper par une porte laissée ouverte ${ }^{36}$, de courir les champs ${ }^{37}$, et celui d'obtenir la grâce, faute de toute possibilité d'échapper.${ }^{38}$ C'est d'ailleurs la dernière chose à laquelle il pense avant de passer à l'échafaud. Pour ce qui est du Baiser de la femmearaignée, la lueur d'espoir n'apparaît que dans les dernières pages où il s'agit du rêve d'Arregui endormi sous l'effet de la morphine : A la fin du tunnel qu'il traverse, il voit une lumière et une femme (la femme-araignée) qui l'aide à sortir de l'eau. De son côté, pour Bossaty, une lueur d'espoir apparait dans les deux petits oiseaux placés à la droite du tableau de Van Gogh, symbolisant ainsi la liberté.

A part la peinture présente dans le paratexte, elle est aussi présente dans l'action : dans Le baiser de la femme-araignée, Irena a 
fait des études de peinture et elle ne dessine que des panthères. Ces panthères dédoublées par Irena rappellent les prisonniers dédoublés de Van Gogh, évoquant aussi l'obsession qui la hante (celle de devenir panthère). D'autre part, la panthère en cage est l'allégorie de Molina dans le cachot (équivalent symbolique de la cage) et, d'ailleurs, son nom évoque l'idée du moulin qui tourne à l'infini. Le dessin apparaît aussi dans Barreaux, évoquant la même valeur ; à savoir reproduire le réel par la métaphore. Les prisonniers de Barreaux dessinent le policier sous la forme d'un chien sauvage avec des canines féroces, ayant un grand couteau en main. ${ }^{39} \mathrm{C}$ 'est ainsi que ce qui est dessiné n'est pas le prisonnier (comme pour Puig) mais le geôlier. Le dessin sert ici à dénoncer l'autorité et à montrer comment elle est vue par le prisonnier. Face à cette désignation qui se fait par l'intermédiaire du dessin, le prisonnier du Dernier jour d'un condamné utilise la métaphore pour désigner les juges, les procureurs du roi et les spectateurs qui ont assisté au procès : «des corbeaux autour d'un cadavre », ${ }^{40}$ par opposition aux pigeons que Salem élève sur le toit qui dénotent l'espoir et qui sont, d'ailleurs, cités dans la première phrase du roman. Un prisonnier demande à Salem s'il élève ces pigeons pour les tuer ; celui-ci répond par la négative en confirmant que ces pigeons lui font oublier l'atrocité du monde. Mais il est à noter qu'à la fin du roman, il ne restait plus que trois pigeons des vingt, vu que Salem ne montait plus sur le toit pour les alimenter. Ces pigeons élevés par Salem s'opposent aux oiseaux en cage qui sont l'allégorie $\mathrm{du}$ prisonnier. Ces oiseaux s'agitent en voyant Irena entrer dans le magasin, tout comme ceux que l'architecte élève chez lui et qui meurent en voyant la femme-panthère.

Une métaphore dominante est à relever; à savoir celle de l'araignée. Symboliquement parlant, il s'agit du geôlier, contrairement à la panthère qui est le prisonnier. Elles s'opposent, de même, aux pigeons de Salem qui connotent la valeur de la liberté. Dans ce contexte, il est à noter que le travail de l'araignée est un travail 
organisé et minutieux, contrairement aux mouvements de la panthère décrits par Molina: celle-ci se meut avec fureur et d'une manière désorganisée. Sur le plan symbolique, le travail organisé rappelle l'architecture (Puig a fait des études d'architecture) régi par des règles spécifiques, alors que les mouvements aléatoires appartiennent à l'état de la nature qui n'est soumis à aucune contrainte ni règle, donc qui se caractérise par la liberté. Le travail de l'araignée consiste à tisser métaphoriquement les barreaux d'une prison vu qu'elle tisse sa toile autour de sa victime graduellement. Par ailleurs, l'araignée revêt une valeur de protection, tel qu'il est cité dans le Coran : sa toile a protégé le Prophète. L'idée de la protection perfide apparaît dans Le dernier jour d'un condamné, mais elle est citée par le biais de l'humour noir : le condamné parle de l'importance de le garder en bonne santé (et en bon état) pour le spectacle sanglant de l'exécution ${ }^{41}$. D'autre part, la toile de l'araignée abrite les noms des condamnés inscrits sur les murs : ce n'est qu'en arrachant cette toile que le condamné les découvre. Cette même araignée l'attaque : «J'ai senti se trainer sur mon pied nu un ventre froid et des pattes velues. C'était l'araignée que j'avais dérangée. $»,{ }^{42}$. Pour ce qui est du Baiser de la femme-araignée, l'araignée n'apparait qu'en métaphore. C'est Arregui qui désigne Molina par «femme-araignée », ${ }^{43}$ : «Tu es la femme-araignée qui attrape les hommes dans sa toile. » Cette métaphore évoque, dans ce cas, la valeur de la protection: Molina (initialement placé dans la cellule pour soutirer des informations d'Arregui) sympathise avec lui et refuse d'assumer la tâche demandée, malgré la pression du procureur. En effet, à plusieurs reprises, Molina demande à son compagnon de cellule de ne rien lui dire sur ses camarades. Ce n'est qu'à la fin du roman qu'Arregui lui parle d'eux.

Outre l'araignée, l'idée est aussi une geôlière par la métaphore. En effet, le condamné du roman français le déclare : «Cette idée fixe qui me possède », ${ }^{44}$ « Mon esprit est en prison dans une idée, une horrible, une sanglante et une implacable idée. $»,{ }^{45}$ Cette idée est 
souvent associée à la glace et au sang. Dans son cas, il s'agit de l'idée de la mort qui est personnifiée, alors que pour Bossaty et pour Puig, il s'agit de détenus politiques emprisonnés pour avoir défendu des idées de gauche. S'y ajoute qu'Irena (Le baiser de la femme-araignée) est prisonnière d'une obsession: elle a peur d'être la descendante des femmes-panthères). A la fin du film intitulé Peuple de chats, elle devient panthère et tue le psychanalyste.

Ces images reflètent toutes un espace hostile d'étouffement qui s'oppose radicalement aux espaces de l'intimité protectrice analysé par Bachelard dans La poétique de l'espace.

Si ces instances narratives ne passent pas sous silence l'aspect social et poétique déjà vus du monde carcéral, elles ne manquent pas de faire coïncider Histoire et fiction comme autre forme de témoignage

\section{L'agencement de l'Histoire et de la fiction}

L'Histoire et la fiction se recoupent dans ces récits qui se basent tous sur l'enchevêtrement et, par la suite, la multiplicité temporelle et la fragmentation, comme nous allons le montrer dans les paragraphes qui suivent.

Les trois auteurs font appel à d'autres époques historiques outre celles qui appartiennent aux contextes historiques de leurs récits. Cependant, l'Histoire est insérée dans la fiction par trois alibis ou médiums distincts : les souvenirs de Salem dans Barreaux, les films racontés par Molina dans Le baiser de la femme-araignée et les inscriptions sur les murs des cachots dans Le dernier jour d'un condamné.

Pour sa part, Barreaux est un récit à la première personne dont la narration est assumée par Salem, le gardien de la prison où sont détenus les prisonniers politiques. Il raconte des histoires relatives à de différents prisonniers politiques qui s'entrecroisent. Ensuite, il 
évoque ses propres souvenirs d'enfance placés dans le contexte de la prison puisqu'il est le fils d'un gardien de prison à la retraite et qui habitait non loin de la prison. Ces souvenirs sont suivis par la période de sa jeunesse (celle du présent de la narration) qui occupe la plus grande part du récit. Ensuite, une brève partie est consacrée à l'époque de sa retraite. C'est ainsi que le roman est basé sur le principe de la fragmentation.

Dans Barreaux, certains événements historiques sont présentés sous forme de souvenirs et d'autres comme faisant partie de l'actualité de la narration, tout comme pour le récit de vie de Salem. Il est à noter que tous les événements historiques cités sont présentés par le biais de l'allusion. De la première série d'événements, nous pouvons en citer deux par ordre chronologique : d'abord, une référence implicite à la déclaration faite par Sadat selon laquelle il n'y aurait plus de camps de détention, par référence à ceux que Nasser avait construits. Cette déclaration date du15 Mai 1971 dans le cadre de la Révolution dite au cours de laquelle Sadat s'est débarrassé des partisans de Nasser considérés comme étant ses ennemis. En effet, après la mort de Nasser, ses partisans étaient divisés en deux groupes : ceux qui favorisaient l'accès de Sadat au pouvoir (vu qu'il était le viceprésident) et ceux qui s'y sont opposés (et par suite séquestrés par Sadat). Bossaty ne cite ni le nom de Sadat ni la date de la démolition du camp de détention. Cependant, il ne manque pas de rappeler par le biais de l'allusion que Sadat a reconstruit ces camps de détention.

Pour ce qui du second événement, il s'agit du naufrage du ferryboat (baptisé Al Salam 98) qui avait eu lieu en 2006 dans la Mer Rouge sous Mobarak. Ce ferry-boat transportait des pèlerins qui revenaient de La Mecque vers l'Egypte. La salle des machines avait pris feu et les canots de sauvetage étaient insuffisants puisqu'il transportait des passagers plus nombreux que le nombre autorisé. Salem parle de corruption et ne manque pas de signaler que les 
coupables n'avaient pas été sanctionnés parce qu'ils étaient pistonnés. L'auteur ne cite pas leurs noms non plus, contrairement aux personnages fictifs qui sont tous nommés.

Pour ce qui est des événements présentés comme faisant partie de l'actualité de la narration, relatés dans Barreaux, ils sont semblables à ceux qui avaient eu lieu sous Nasser et Sadat, mais Salem ne nomme pas explicitement les deux dirigeants politiques. Parmi les événements historiques cités qui datent de cette époque, nous citons la détention de journalistes de l'opposition qui dénonçaient la corruption du gouvernement et la torture des prisonniers politiques. Toutefois, le nom de Mostafa Amin ${ }^{46}$ est cité par le prisonnier envoyé pour agresser le journaliste. L'actualité de la narration devrait se placer à partir de 2006, puisque le naufrage du ferry-boat (qui avait eu lieu en 2006) est présenté sous forme de souvenir. De même, Salem parle d'un groupe de «révolutionnaires qu'on venait de détenir et qui devraient être libérés deux semaines plus tard», ${ }^{47}$, «Les deux semaines se sont écoulées, l'année s'est achevée, et ils sont toujours détenus. $»^{48}$ Il signale qu'ils sont nombreux, de sorte que leur nombre dépasse celui des gardiens. Il ne donne aucune information précise sur ces événements.

Tout comme Bossaty, Puig ne cite que tardivement des événements historiques qui appartiennent à l'actualité de la narration, et plus précisément au septième chapitre. Cependant, il situe son récit explicitement dans le temps sans allusion: En effet, Le baiser de la femme-araignée se déroule dans l'Argentine des années soixante-dix. Selon les rapports de la police, la sentence de condamnation de Molina a été émise le 20/7/1974 à Buenos Aires et il a été transféré à la cellule où Arregui était emprisonné le 4/4/1975. Ce dernier a été arrêté le 16/10/1972 puisqu'il était à la tête de la grève ouvrière. En outre, il a mené la grève de faim en prison, suite à la mort d'un prisonnier politique (Juan Vicente Aparicio) durant les interrogations policières 49 
A part le contexte de l'Argentine des années soixante-dix, d'autres références à l'Histoire sont citées. Ces références ne sont pas placées sous forme de souvenirs comme pour Bossaty, mais elles sont présentées comme servant de cadre aux films racontés par Molina à Arregui. Tout comme le contexte du récit-cadre, ces deux périodes sont des périodes de tumultes politiques. C'est pourquoi le choix des films racontés n'est pas arbitraire. Il est à remarquer, dans ce contexte, que dans tous les films racontés, il y a toujours une clé perdue. Cet élément est à mettre en rapport avec le thème de la prison puisque, symboliquement, il s'agit de la clé d'une prison : à la fois ouvrir (la liberté) et fermer (l'aliénation). D'autre part, le personnage principal (la panthère et la chanteuse) meurent écrasés par une automobile, tout comme Molina qui tombe après avoir reçu une balle tirée à partir d'une automobile. Un troisième élément répétitif en rapport avec le thème de la prison : un personnage se sent poursuivi.

Pour ce qui est du premier événement historique, il est cité dans le film intitulé Peuple de Chats (1942). Irena, l'héroïne, est une réfugiée à New York à cause de la guerre. Bien que Molina ne précise pas de quelle guerre s'agit-il (voire il se contente de dire qu'elle vient de la montagne), dans le film initial il est question de l'invasion mamelouk de la Transylvanie qui avait eu lieu au XIIIème siècle. Dans ce même film, une référence à la "guerre sainte » est citée dans le cadre d'une légende relative à la femme-panthère.

Pour ce qui est du second évènement, il sert de cadre au film intitulé Destin. C'est un film qui défend l'idéologie nazie et, par la suite, provoque la colère d'Arregui, le militant de gauche. L'histoire se déroule sous l'occupation nazie de la France datant de la Deuxième Guerre Mondiale, tel que le déclare Molina. Léni Lamaison, la célèbre chanteuse française, tombe amoureuse d'un leader fasciste allemand. Elle le trahit en lui soutirant des informations, mais elle trahit aussi son propre pays en se laissant contaminer par le racisme national 
socialiste. Elle meurt pour la cause politique de son amant, tout comme Molina qui meurt pour la cause d'Arregui. C'est ainsi que des rapports peuvent être établis entre ce film et le récit initial.

Pour ce qui est du dernier jour d'un condamné, il se compose de trois phases principales réparties selon l'espace : la prison de Bicêtre, le transfert à la conciergerie (c'est la période qui se termine par le refus du pourvoi), le transfert à l'Hôtel de Ville (c'est la période qui se termine sur l'exécution sur laquelle se clôt le roman). Ces trois lieux sont des symboles historiques témoignant de l'atrocité de la peine de mort. Outre ces témoignages spatiaux sur des époques révolues, Hugo insère dans la fiction des personnalités historiques mais qui sont reléguées au second plan de l'action principale, contrairement à Puig. En effet, les prisonniers des romans égyptien et argentin sont des prisonniers politiques de l'opposition, alors que celui de Hugo est l'auteur d'un meurtre qu'il ne cherche pas à nier bien qu'il ne soit pas prémédité ${ }^{50}$. Rien n'est cité sur le crime commis par le héros, mais un épisode permet au lecteur de le deviner : il s'agit du meurtre de la vieille qui s'était introduite clandestinement chez le héros alors qu'il était en train de jouer aux cartes avec ses compagnons. Deux types de personnalités historiques qui ont réellement existé sont introduits via l'inscription sur les murs sombres des cachots une fois éclairés par la lumière de la lanterne: des assassins et des prisonniers politiques guillotinés pour leur militantisme de gauche. Pour ce qui est des assassins, nous citons Papavoine (exécuté en 1825 pour le meurtre injustifié de deux enfants), Dautun (exécuté en 1815 pour avoir tué son frère), Poulain (exécuté en 1818 pour avoir assassiné sa femme), Jean Martin (exécuté en 1821 pour avoir assassiné son père) et Castaing (un médecin qui avait empoisonné son ami en 1823). Pour ce qui est des inscriptions concernant les prisonniers politiques, deux éléments contradictoires sont relevés : «Vive l'Empereur! 1824 » et «La République », visant ainsi à souligner la généralisation de la guillotine. La mention «La République » apparaît près du nom de 
Bories, un des quatre sous-officiers de la Rochelle (les trois autres sont Goubin, Pommier et Raoulx). Ces quatre sous-officiers étaient contre la royauté et la restauration des Bourbons et ils appartenaient au mouvement désigné par «la charbonnerie», une organisation clandestine qui militait contre la Restauration en cherchant à fonder une «assemblée constituante » basée sur la souveraineté du peuple. Ces quatre sous-officiers ont été exécutés en 1822. D'autre part, les noms de Robespierre et de Louis XVI (morts guillotinés) sont cités par le gardien. Il est à remarquer que les personnages historiques sont tous désignés par leurs noms, alors que le héros (le personnage fictif) est anonyme (contrairement à ce que Bossaty et Puig font puisqu'ils gardent l'anonymat pour les personnages historiques). Toutes ces personnalités (les criminels et les prisonniers politiques) partagent le même destin du héros: la mort par la guillotine. C'est ainsi que l'Histoire converge avec la fiction qui sert d'alibi pour dénoncer ces pratiques généralisées de la guillotine à laquelle étaient destinés ceux qui s'opposaient au pouvoir.

De ce qui précède nous pouvons conclure que, pour les trois auteurs, les époques historiques cadrant les récits sont une autre facette de celles qui sont citées et placées en marge du récit initial : Pour Bossaty, le cadre historique de la narration (situé après 2006) converge avec les événements historiques ayant lieu sous Nasser et Sadat. De son côté, pour Puig, les films racontés mettent en évidence eux-aussi une autre forme de dictature militaire. Pour Hugo, les personnalités historiques (assassins ou prisonniers politiques) partagent le même destin que celui du héros.

Le désir de changer le cours de l'Histoire par l'écriture apparaît chez Hugo et Bossaty. En effet, le condamné de Hugo formule le désir d'écrire un journal qui serait un témoignage historique sur les souffrances que subissent les condamnés à mort. Il raconterait les angoisses et la terreur qui les obsèdent et que personne 
ne connaît, dans le but d'émouvoir et de lutter contre les atrocités de la peine de mort. Il lui donnerait pour titre «l'autopsie intellectuelle d'un condamné », 51, donc il s'agirait d'une écriture fondée sur l'introspection. Il cherche ainsi à sauver les autres de ce destin fatal et à changer le cours de l'Histoire surtout à une époque où le nombre de guillotinés est en croissance. Ce journal n'est autre que le projet de Hugo réalisé par ce roman, puisqu'il s'agit de dévoiler un aspect souvent gardé sous silence de la peine de mort. Face à ce journal d'un condamné qui cherche à dénoncer la torture morale que les condamnés à mort subissent, les prisonniers dans Barreaux publient un journal où sont dénoncées les atrocités des prisons et les mauvais traitements qu'ils subissent. De même, ils y critiquent la politique du gouvernement, entre autres la hausse des prix, l'argent dans les banques étrangères, la corruption des députés et la répression qu'exerce 1'Etat ${ }^{52}$. Mais ce journal est déchiré par l'autorité dès que les prisonniers l'accrochent au mur. En outre, Salem évoque la détention d'un personnage présenté comme étant un professeur universitaire et auteur d'un ouvrage intitulé غباء الاستبداد. Ce titre est une parodie de celui de Kawakbi intitulé طبائع الاستبدادو مصارع الاستعباد الاستراد dont l'auteur critique toute forme de répression tout en montrant comment s'y opposer. Kawakbi a lutté contre la corruption des gouverneurs ottomans et, par la suite, il est mort empoisonné étant donné que les tentatives pour le tuer n'avaient pas abouti. C'est ainsi que Bossaty fait le parallèle entre deux cas d'intellectuels détenus pour leurs idées. En outre, Salem cite aussi le cas d'un économiste qui avait publié des recherches sur l'effondrement économique (nommé Maurice, sans nom de famille) dénonçant ainsi la corruption et, par suite, il a été séquestré, ${ }^{53}$. Cette tentative de dénoncer l'autorité (sur plusieurs plans) comme témoignage historique est donc vouée à l'échec, tout comme celle du condamné (Le dernier jour d'un condamné) qui cherchait à dénoncer cet aspect sanglant de l'Histoire.

Dans ce même contexte, une même question épineuse et 
problématique en rapport avec la prison est soulevée par les trois auteurs; à savoir: pour ou contre l'engagement pour une cause politique ou une idée? En d'autres mots: pour ou contre un Prométhée voleur de feu pour sauver l'humanité ? Toutefois, les personnages ne partagent pas la même réponse. Le héros du roman de Hugo formule des réflexions à propos des prisonniers politiques ; réflexions dont l'essentiel réside dans la condamnation de ce qu'il appelle «nécessités politiques » ${ }^{54}$ et le refus de l'idée de mourir pour une cause. Cette même idée apparaît chez Bossaty : le prisonnier (il s'agit d'un criminel et non d'un prisonnier politique) envoyé pour agresser le journaliste de gauche dit à ce dernier qu'il condamne le fait de militer ou de s'engager pour une cause, ${ }^{55}$. Cette pensée s'oppose complètement à celle d'Arregui dont l'idéal est le marxisme pour lequel il sacrifie sa vie. En effet, sa vie privée n'est que secondaire : «Je vis en fonction d'une lutte ou d'une activité politique », ${ }^{56}$ étant donné qu'il se consacre entièrement à une cause noble: «La révolution sociale ${ }^{57}$. Rien d'étonnant si, dans le langage codé des correspondances entre les membres du mouvement marxiste auquel il appartient, « la lutte pour la cause » est désignée par «la lutte pour la vie ${ }^{58}$

En guise de conclusion, nous soulignons la dimension mythologique de l'espace hostile qu'est la prison. En effet, elle est le symbole de la caverne primordiale considérée comme le monde des ombres dans la mesure où elle permet de voir le réel différemment. Il s'agit d'un réel qui dépasse la pensée immédiate de l'homme et qui porte sur les notions de vie et de mort. En effet, cet espace est envisagé comme espace transitoire entre la vie et la mort, donc un espace de résurrection où une sorte de catharsis a lieu. Il s'agit de la purification de l'âme de tous les péchés.

D'autre part, la caverne est considérée comme l'archétype primordial qui symbolise le retour à la terre-mère et aux origines liées 
aux profondeurs, par opposition à la montagne liée aux valeurs archétypales de la sublimation. C'est pourquoi, dans les théories de la psychanalyse, la caverne est le symbole de l'inconscient où l'essence de la psyché humaine réside.

Dans les récits de prison, la caverne, symbolisée par le cachot, est envisagée comme espace d'hostilité et elle génère une angoisse claustrophobique qui crée un sentiment intense d'étouffement. $\mathrm{Ce}$ sentiment se fraye une voie à travers l'aspect social et poétique de ces récits hétérogènes où l'Histoire et la fiction sont combinés pour exprimer une autre facette de l'Histoire vouée à l'ombre de l'Histoire officielle. 


\section{Notes :}

1 Bachelard, Gaston, La poétique de l'espace, Presses Universitaires de France, Paris, 1957, p.17

2 Padis, Marc Olivier, Une littérature de l'enfermement, Dans : Esprit, n : 226, publiée par Editions Esprit, novembre 1994, pp.114-125. URL stable : https//www.jstor.org/stable/24276333

3 Tomiche, Nada, La littérature arabe contemporaine, collection Orient, éditions Maisonneuve et Larose, Paris, 1993, introduction p.7.

4 Cuervo Hewitt, Julia, El texto ausente en El beso de la mujer araña de Manuel Puig : Silencios y reticencias de una época (Le texte absent dans Le baiser de la femme-araignée de Manuel Puig : Silences et réticences d'une époque), Dans Chasqui, revue de littérature latino-américaine, volume 19, n: 2, novembre 1990, pp.51-57. URL stable : https//www.jstor.org/stable/29740273. L'article a été lu dans sa version originale en espagnol.

5 Naudin, Marie, Hugo et Camus face à la peine capitale, Dans : Revue d'Histoire littéraire de la France, 72ème année, n : 12, publiée par Presses universitaire de France, Mars-Avril 1972, pp.264-273

6 Guillén, Claudio, Entre lo uno y lo diverso ; introducción a la literatura comparada (Entre l'Un et le Divers, introduction à la littérature comparée), Tusquet, Barcelone, 2005, p.39. La citation a été traduite par le chercheur.

7 Pageaux, Daniel-Henri, La littérature générale et comparée, Armand Colin, Paris, 1994, p.93

8Lukacs, Georg, La théorie du roman, traduit de l'allemand par Jean Clairevoye, Gallimard, Paris, 1968, p.31

9 De façon générale, les récits de Mohammed Al Bossaty traitent tous, d'une manière ou d'une autre, de la marginalisation. Sa production littéraire est nombreuse; nous en citons des romans comme Les voix de la nuit (1998), Le train arrive (1999) et Famine (2005). Il a remporté le premier prix du concours du meilleur roman organisé lors de la foire internationale du livre au Caire en 1994 pour son roman intitulé Le bruit du lac.

10 Manuel Puig est un des écrivains argentins les plus lus. Parmi ses romans les plus connus, nous citons : La trahison de Rita Hayworth (1968), L'affaire de Buenos Aires (1973) et Le baiser de la femme-araignée (1976) qui a été censuré juste après sa publication. 
11 Le péronisme est un mouvement politique argentin nommé d'après le colonel Juan Domingo Perón (1895-1974), président de l'Argentine pour trois mandats : de 1946 à 1952, de 1952 à 1955 et de 1973-1974. Ses idées sont recensées sous forme de vingt vérités dont la plus importante se base sur la démocratie comme volonté du peuple et sur les droits des travailleurs et la justice sociale. Plus tard, le péronisme s'est divisé en deux partis politiques : le parti justicialiste (de la gauche) qui a respecté la politique de Perón et le péronisme de droite (qui défendait l'idée du « rétrécissement de l'Etat» inspirée du néolibéralisme. Par « néolibéralisme », Il s'agit d'un mouvement idéologique issu du libéralisme qui visait à limiter l'intervention de l'Etat dans les questions économiques. Ce mouvement a pris naissance après la Première Guerre Mondiale.

12 Son œuvre revêt souvent un aspect politique. Dans Les misérables par exemple, il parle de l'injustice sociale qui caractérisait la France du XXème siècle. D'autre part, il est l'auteur de pièces de théâtre, de romans et de poésie. Parmi ses romans les plus célèbres, nous citons Notre Dame de Paris (1831) et Les misérables (1862). De ses recueils poétiques, nous citons Les Contemplations (1856) et Les Châtiments (1853) et de ses pièces de théâtre Cromwell (1827) et Hernani (1830).

13 Hugo, Victor, Le dernier jour d'un condamné (de p.61 à p.160), suivi de Claude Gueux et de L'affaire Tapner (1829), Le livre de Poche, Paris, 1989, p.76

14 Les informations scientifiques placées sous la forme de notes de bas de page ayant comme sujet l'homosexualité, le rapport scientifique de la police concernant Molina et Arregui qui interrompt brusquement le fil de l'action sans être introduit formellement et une note documentaire sur la chanteuse Léni Lamaison comme personnalité historique.

ه البساطى ، محمد ، أسورار ، دار أخبار اليوم ، قطاع الثقافة ، جمهورية مصر العربية ، ^ . . ب ، صفحة

المرجع السابق ، صفحة بr 16

المرجع السابق ، صفحة 9 ؛ 17

18 Puig, Manuel, El beso de la mujer araña (1976), (Le baiser de la femmearaignée), Bibliothèque de Bolsillo, Espagne, 1990, pp.65-67

19 Hugo, Victor, Le dernier jour d'un condamné (de p.61 à p.160), suivi de Claude Gueux et de L'affaire Tapner (1829), op.cit., p.81

20 Ibid, pp.108-109

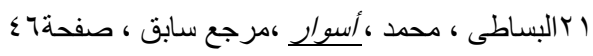


22 Hugo, Victor, Le dernier jour d'un condamné (de p.61 à p.160), suivi de Claude Gueux et de L'affaire Tapner (1829), op.cit., p.118

23 Puig, Manuel, El beso de la mujer araña (1976), (Le baiser de la femmearaignée), op.cit, p.190

24 Bakhtine, Mikhail, Esthétique et théorie du roman, Gallimard, Paris, 1978.

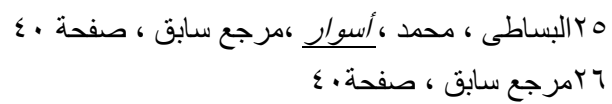

27 Puig, Manuel, El beso de la mujer araña (1976), (Le baiser de la femmearaignée), op.cit, p.98

28 Hugo, Victor, Le dernier jour d'un condamné (de p.61 à p.160), suivi de Claude Gueux et de L'affaire Tapner (1829), op.cit., p.121

29 Hugo, Victor, Le dernier jour d'un condamné (de p.61 à p.160), suivi de Claude Gueux et de L'affaire Tapner (1829), op.cit, pp.148-153

30 Ibid, p.85

31 Ibid, p.121

32 Ibid p.129-130

33 Ibid, p.102

34 Ibid, p.77

35 Ibid, p.78

36 Ibid, p.118

37 Ibid, p.126

38 Ibid, p.119

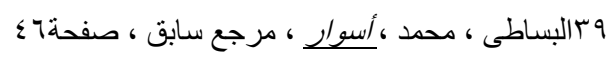

40 Hugo, Victor, Le dernier jour d'un condamné (de p.61 à p.160), suivi de Claude Gueux et de L'affaire Tapner (1829), op.cit., p.7

41 Hugo, Victor, Le dernier jour d'un condamné (de p.61 à p.160), suivi de Claude Gueux et de L'affaire Tapner (1829), op.cit., p.86

42 Ibid, p. 100

43 Puig, Manuel, El beso de la mujer araña (1976), (Le baiser de la femmearaignée), op.cit, p.81

44 Hugo, Victor, Le dernier jour d'un condamné (de p.61 à p.160), suivi de Claude Gueux et de L'affaire Tapner (1829), op.cit. p.86

45 Ibid, p.70 
46 Mostafa Amin est un journaliste libéral (1914-1997). Il est le neveu de Saad Zaghloul, une des figures politiques les plus éminentes de l'Histoire égyptienne, et le fondateur du parti libéral nationaliste. Mostafa Amin est également le fondateur du quotidien Akhbar Al Yom et de la revue Rose Al Youssef. Il défendait la liberté de l'expression et il a été séquestré par Nasser en 1950 et par Sadat en 1974.

99 § V البساطى ، محمد ، أسور ، مرجع سابق ، صفحة

48 Ibid

49 Puig, Manuel, El beso de la mujer araña (1976), (Le baiser de la femmearaignée), op.cit, p.104

50 Hugo, Victor, Le dernier jour d'un condamné (de p.61 à p.160), suivi de Claude Gueux et de L'affaire Tapner (1829), op.cit, p.77

51 Ibid, p.73

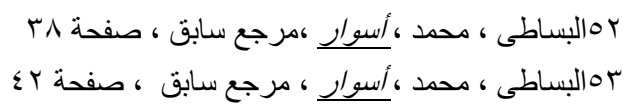

54 Hugo, Victor, Le dernier jour d'un condamné (de p.61 à p.160), suivi de Claude Gueux et de L'affaire Tapner (1829), op.cit, p.109

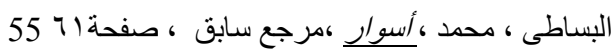

56 Puig, Manuel, El beso de la mujer araña (1976), (Le baiser de la femmearaignée), op.cit, p.22

57 Ibid p. 22

58 Ibid, p.95 


\section{Bibliographie}

\section{Corpus :}

1. Hugo, Victor, Le dernier jour d'un condamné (de p.61 à p.160), suivi de Claude Gueux et de L'affaire Tapner (1829), Le livre de Poche, Paris, 1989.

2. Puig, Manuel, El beso de la mujer araña (1976), (Le baiser de la femme-araignée), Bibliothèque de Bolsillo, Espagne, 1990. Toutes les citations ont été traduites par le chercheur.

r. البساطى ، محمد ، أسوار ، دار أخبار اليوم ، قطاع الثقافة ، جمهورية مصر العربيـة ،

\section{Ouvrages généraux :}

1. Bachelard, Gaston, La poétique de l'espace, Presses Universitaires de France, Paris, 1957

2. Bakhtine, Mikhail, Esthétique et théorie du roman, Gallimard, Paris, 1978.

3. Durand, Gilbert, Les structures anthropologiques de l'imaginaire, Dunod, Paris, 1992

4. Guillén, Claudio, Entre lo uno y lo diverso ; introducción a la literatura comparada (Entre l'Un et le Divers, introduction à la littérature comparée), Tusquet, Barcelone, 2005.

5. Lukacs, Georg, La théorie du roman, traduit de l'allemand par Jean Clairevoye, Gallimard, Paris, 1968

6. Pageaux, Daniel-Henri, La littérature générale et comparée, Armand Colin, Paris, 1994

7. Tomiche, Nada, La littérature arabe contemporaine, collection Orient, éditions Maisonneuve et Larose, Paris, 1993

\section{Articles de revues sur les auteurs et les romans :}

\section{En espagnol :}

1. Bianchi, Soledad, Manuel Puig : la tentación de espiar, (Manuel Puig: La tentation d'espionner), Dans: Revue Chilienne de 
littérature, n: 38, publiée par l'université du Chili, novembre 1991, pp.109-113. URL stable :

https://www.jstor.org/stable/40356625

2. Coddou, Marcelo, Complejidad estructural de El beso de la mujer araña de Manuel Puig, (Complexité structural du Baiser de la femme-araignée de Manuel Puig), Dans : Revue de la littérature hispanique, $\mathrm{n}: 7$, publiée par INTI, printemps 1978, pp.15-27. URL stable : https://www.jstor.org/stable/23285008

3. Cuervo Hewitt, Julia, El texto ausente en El beso de la mujer araña de Manuel Puig : Silencios y reticencias de una época, (Le texte absent du baiser de la femme-araigée de Manuel Puig : Silences et réticences d'une époque, Dans Chasqui : Revue de littérature latino-américaine, vol. 19, publiée par Chasqui, pp.5157. URL stable : https://www.jstor.org/stable/29740273

4. Fabry, Geneviève, Las aporías de la visión en la novelística de Manuel Puig, (Les apories de la vision dans les romans de Manuel Puig), Dans : Revue Chilienne de littérature, $\mathrm{n}$ : 51, publiée par l'université du Chili, novembre 1997, pp.28-38. URL stable : https://www.jstor.org/stable/40356893

5. Marta Fernandez, Liliane, Manuel Puig, El verso y el reverso del texto (Manuel Puig, le vers et le revers du texte), Dans: Hispanoamérica n : 50, publiée par Saul Sosnowski, Août 1988, pp.47-57

\section{En francais :}

1. Comeau, Paul, La rhétorique du poète engagé du Dernier jour d'un condamné à Claude Gueux, Dans: Etudes françaises du XIXème siècle, vol.16, n: 1/2, publiée par les presses de l'université de Nebraska, automne / hiver 1987-1988, pp.59-77. URL stable : https://www.jstor.org/stable/23532082

2. Lowe-Dupas, Hélène, Innommable guillotine : la peine de mort dans Le dernier jour d'un condamné et Histoire d'Hélène Gillet, 
Dans: Etudes françaises du XIXème siècle, vol.23, n: 3/4, publiée par les presses de l'université de Nebraska, printemps été 1995, pp.341-348. URL stable : https://www.jstor.org/stable/23537062

3. Malavié, Jean, A propos du Dernier jour d'un condamné : Nodier ou Nisard, critique de Victor Hugo ? Dans : Revue d'Histoire littéraire de la France, n: 3 , publiée par les presses de l'université de France, Mai-Juin 1993, pp.446-450. URL stable : https://www.jstor.org/stable/40527468

4. Naudin, Marie, Hugo et Camus face à la peine capitale, Dans : Revue d'Histoire littéraire de la France, n: 2 , publiée par les presses de l'université de France, Mars-Avril 1972, pp.264-273. URL stable : https://www.jstor.org/stable/40524272

5. Padis, Marc Olivier, Une littérature de l'enfermement, Dans : Esprit, n : 226, publiée par Editions Esprit, novembre 1994, pp.114-125. URL stable : https//www.jstor.org/stable/24276333 